\title{
RENORMING FOR ELASTIC SYSTEMS WITH STRUCTURAL DAMPING
}

\author{
Richard H. Fabiano* \\ Department of Mathematical Sciences \\ 340 Bryan Building \\ University of North Carolina at Greensboro \\ Greensboro, NC 27412 \\ fabianoQuncg.edu
}

\begin{abstract}
We consider a model of an elastic system with so-called structural damping. The operator associated with such models is known to generate an analytic semigroup, but it is neither sectorial nor associated with a coercive sesquilinear form. We show that these properties can be obtained by using an appropriate inner product other than the usual energy inner product.
\end{abstract}

\section{INTRODUCTION}

We consider the following abstract model of an elastic system with damping, which is a second order differential equation on a Hilbert space $X$ :

$$
\begin{gathered}
\ddot{u}(t)+A u(t)+2 a A^{1 / 2} \dot{u}(t)=0, \\
u(0)=u_{0}, \quad \dot{u}(0)=v_{0} .
\end{gathered}
$$

Here we assume that $A$ is a positive definite, self adjoint unbounded linear operator on $X$, with compact inverse, and with a positive definite, self adjoint square root $A^{1 / 2}$. It is standard to reformulate (1.1) on the energy space $H=$ $\operatorname{dom} A^{1 / 2} \times X$ equipped with the energy norm $\|(u, v)\|_{H}^{2}=\left\|A^{1 / 2} u\right\|_{X}^{2}+\|v\|_{X}^{2}$. If we identify $z(t)=(u(t), \dot{u}(t))$, then (1.1) can be reformulated as the first order system

$$
\begin{aligned}
\dot{z}(t) & =\mathcal{A} z(t) \\
z(0) & =\left(u_{0}, v_{0}\right) .
\end{aligned}
$$

Here $\mathcal{A}$ is defined on the domain

$$
\operatorname{dom} \mathcal{A}=\left\{(u, v) \in H: v \in \operatorname{dom} A^{1 / 2}, u \in \operatorname{dom} A\right\}
$$

*This research was supported in part by the National Science Foundation under grant DMS9696239.

The original version of this chapter was revised: The copyright line was incorrect. This has been corrected. The Erratum to this chapter is available at DOI: 10.1007/978-0-387-35359-3_40 
by $\mathcal{A}(u, v)=\left(v,-A u-2 a A^{1 / 2} v\right)$. It is well known that the closure of $\mathcal{A}$ is the infinitesimal generator of an analytic semigroup $T(t)$ on $H$ (see [3], [4], [7], and [10]). However, the operator $-\mathcal{A}$ is neither sectorial nor associated with a coercive form. In this paper we show how to use an equivalent inner product to obtain these properties. Let us first recall some relevant definitions and results.

For an operator $A$ defined in a Hilbert space $H$, the numerical range of $A$ (also called the field of values of $A$ ), denoted by $\Theta(A)$, is the subset of the complex plane defined by $\Theta(A)=\left\{\langle A x, x\rangle_{H}: x \in \operatorname{dom} A,\|x\|_{H}=1\right\}$. The numerical range is inner product dependent, and sometimes this is indicated explicitly by the notation $\Theta(A,\langle\cdot, \cdot\rangle)$. The numerical range is convex [8] and always contains the eigenvalues, and hence contains the convex hull of the eigenvalues. The closed operator $A$ is m-sectorial (with vertex at the origin) if the numerical range $\Theta(A)$ is contained in a sector of the form $|\arg (\lambda)| \leq$ $\theta<\pi / 2$ and if $\left\|(\lambda I-A)^{-1}\right\| \leq 1 /(-\operatorname{Re} \lambda)$ for $\operatorname{Re} \lambda<0$. We say that $A$ is quasi $\mathrm{m}$-sectorial if a translation $A+k I$ is $\mathrm{m}$-sectorial. Next, given Hilbert spaces $V \subset H$, with the embedding dense and continuous, the sesquilinear form $\sigma: V \times V \rightarrow \mathbb{C}$ is bounded if there exists $K>0$ such that

$$
|\sigma(x, y)| \leq K\|x\|_{V}\|y\|_{V} \quad \text { for all } x, y \in V
$$

is $V$-elliptic if there exists $c>0$ such that

$$
\operatorname{Re} \sigma(x, x) \geq c\|x\|_{V}^{2} \quad \text { for all } x \in V,
$$

and is $V$-coercive if there exists $c, k \in \mathbb{R}, c>0$, such that

$$
\operatorname{Re} \sigma(x, x) \geq c\|x\|_{V}^{2}-k\|x\|_{H}^{2} \quad \text { for all } x \in V .
$$

In either case $\sigma$ uniquely defines an operator $A: \operatorname{dom} A \subset H \rightarrow H$ by

$$
\sigma(x, y)=\langle A x, y\rangle
$$

for all $x \in \operatorname{dom} A$ and $y \in V$, and

$$
\operatorname{dom} A=\left\{x \in V:|\sigma(x, y)| \leq K_{x}\|y\|_{H} \text { for all } y \in V\right\}
$$

where $K_{x}$ depends on $x$. It is known that if $\sigma$ is $V$-elliptic (resp. $V$-coercive), then the unique operator $A$ defined by $\sigma$ is m-sectorial (resp. quasi m-sectorial). Also, given any quasi m-sectorial operator $A$ (whether or not it is defined by a sesquilinear form as above), it is known that $-A$ is the infinitesimal generator of an analytic semigroup. However, the converse is not true. Thus it is possible for $-A$ to generate an analytic semigroup even though $A$ is not quasi m-sectorial and so does not 'come from' any $V$-coercive form. This is unfortunate, because there are some nice convergence results available for parameter estimation and LQR problems associated with coercive forms (see [2], [1], [9]), especially when the embedding $V \subset Z$ is compact. On the other hand, in such a case we are motivated to seek a new inner product in which $A$ becomes m-sectorial and possibly associated with a $V$-coercive form. This is precisely the situation for 
the model considered in this paper. We refer the reader to $([8],[11],[12],[13])$ for the definitions and results in this paragraph.

In the next section we show how the non-sectorial operator associated with the structural damping model can be made sectorial. Moreover we will see that the operator also becomes associated with a coercive sesquilinear form in the new inner product, which is not possible in the original inner product. Finally, we will see that Galerkin approximations constructed with the new inner product exhibit improved stability robustness compared to approximations in the original inner product.

\section{ELASTIC SYSTEM WITH STRUCTURAL DAMPING}

Consider again equation (1.1). Let $\left\{\phi_{i}\right\}_{i=1}^{\infty}$ denote the eigenvectors of $A$ which form an orthonormal basis for $X$, with corresponding eigenvalues $\left\{\gamma_{i}\right\}_{i=1}^{\infty}$ satisfying $0<\gamma_{1}<\cdots<\gamma_{n} \rightarrow \infty$. We assume that the damping coefficient satisfies $0<a<1$. Physically this is equivalent to assuming that the system is not overdamped. In particular, when $a \geq 1$ then all eigenvalues of $\mathcal{A}$ are negative real numbers (every vibratory mode is overdamped), whereas for $0<a<1$ the spectrum of $\mathcal{A}$ consists of eigenvalues given by

$$
\lambda_{n}^{ \pm}=\alpha_{ \pm} \gamma_{n}^{1 / 2}, \quad \text { where } \alpha_{ \pm}=-a \pm \sqrt{1-a^{2}} i .
$$

Thus the eigenvalues lie on the lines $\operatorname{Im} \lambda=\frac{ \pm \sqrt{1-a^{2}}}{a} \operatorname{Re} \lambda$, and also satisfy $\operatorname{Re} \lambda \leq$ $-a \gamma_{1}^{1 / 2}$.

It is possible to associate $-\mathcal{A}$ with a sesquilinear form, and the standard way to do this is to set $V=\operatorname{dom} A^{1 / 2} \times \operatorname{dom} A^{1 / 2}$ with norm $\|(u, v)\|_{V}^{2}=$ $\left\|A^{1 / 2} u\right\|_{X}^{2}+\left\|A^{1 / 2} v\right\|_{X}^{2}$. Then $V \subset H$ and the embedding is continuous. Also define the sesquilinear form $\sigma: V \times V \rightarrow \mathbb{C}$ by

$$
\sigma((u, v),(f, g))=-\left\langle A^{1 / 2} v, A^{1 / 2} f\right\rangle_{X}+\left\langle A^{1 / 2} u, A^{1 / 2} g\right\rangle_{X}+2 a\left\langle A^{1 / 2} v, g\right\rangle_{X} .
$$

Then $\operatorname{dom} \mathcal{A} \subset V \subset H$ and we have

$$
\langle-\mathcal{A} x, y\rangle_{H}=\sigma(x, y) \text { for all } x \in \operatorname{dom} \mathcal{A}, y \in V .
$$

At this point we note that while $\sigma$ is $V$-bounded, it is neither $V$-elliptic nor $V$-coercive, and the embedding $V \subset H$ is not compact. In fact, it is impossible to associate $-\mathcal{A}$ with a coercive form in this problem. That is, there does not exist a space $V$ continuously embedded in $H$ and a coercive form $\sigma: V \times V \rightarrow \mathbb{C}$ for which $\operatorname{dom} \mathcal{A} \subset V$ and (2.1) holds. The reason is that if there were such a space $V$ and coercive form $\sigma$, then as noted in the introduction it would follow that the numerical range $\Theta(-\mathcal{A})$ is contained in a sector in the complex plane. However, in the energy norm it can be shown that $\Theta(-\mathcal{A})$ is the whole right half complex plane (see [5]). Thus we are motivated to seek a new inner product on $H$, hopefully with the property that the operator $-\mathcal{A}$ in this new inner product is m-sectorial. It turns out that we can achieve the best possible outcome - an 
inner product in which the numerical range $\Theta(-\mathcal{A})$ is equal to the convex hull of the eigenvalues. Furthermore, we can find a space $V$ compactly embedded in $H$, on which there is defined a $V$-elliptic sesquilinear form associated with $-\mathcal{A}$.

To proceed, define the norm $\|\cdot\|_{e}$ on $H$ by

$$
\|(u, v)\|_{e}^{2}=\frac{1}{2\left(1-a^{2}\right)}\left\{\left\|A^{1 / 2} u\right\|_{X}^{2}+\|v\|_{X}^{2}+2 a \operatorname{Re}\left\langle A^{1 / 2} u, v\right\rangle_{X}\right\} .
$$

It is easy to verify that this is a norm which is equivalent to the energy norm, and which has a compatible inner product given by

$$
\begin{gathered}
\langle(u, v),(f, g)\rangle_{e}=\frac{1}{2\left(1-a^{2}\right)}\left\{\left\langle A^{1 / 2} u, A^{1 / 2} f\right\rangle_{X}+\langle v, g\rangle_{X}+a\left\langle A^{1 / 2} u, g\right\rangle_{X}\right. \\
\left.+a\left\langle v, A^{1 / 2} f\right\rangle_{X}\right\} .
\end{gathered}
$$

While it is true that similarly structured quadratic Liapunov functions have been used before for second order damped systems (for example, [6]), it is this precise form which is needed to shrink the numerical range down to the convex hull of the eigenvalues. This is shown in the following theorem.

Theorem The numerical range $\Theta\left(\mathcal{A},\langle\cdot, \cdot\rangle_{e}\right)$ is the convex hull of the eigenvalues of $\mathcal{A}$.

Proof: We must show that $\operatorname{Re}\langle\mathcal{A} x, x\rangle_{e} \leq-a \gamma_{1}^{1 / 2}\|x\|_{e}^{2}$ and $\left|\operatorname{Im}\langle\mathcal{A} x, x\rangle_{e}\right| \leq$ $-\frac{\sqrt{1-a^{2}}}{a} \operatorname{Re}\langle\mathcal{A} x, x\rangle_{e}$ for all $x \in \operatorname{dom} \mathcal{A}$. For $\bar{x}=(u, v) \in \operatorname{dom} \mathcal{A}$ we have

$$
\begin{aligned}
\langle\mathcal{A}(u, v),(u, v)\rangle_{e}= & \left\langle\left(v,-A u-2 a A^{1 / 2} v\right),(u, v)\right\rangle_{e} \\
= & \frac{1}{2\left(1-a^{2}\right)}\left\{\left\langle A^{1 / 2} v, A^{1 / 2} u\right\rangle_{X}+\left\langle-A u-2 a A^{1 / 2} v, v\right\rangle_{X}\right. \\
& \left.\quad+a\left\langle A^{1 / 2} v, v\right\rangle_{X}+a\left\langle-A u-2 a A^{1 / 2} v, A^{1 / 2} u\right\rangle_{X}\right\} \\
= & \frac{1}{2\left(1-a^{2}\right)}\left\{2 i \operatorname{Im}\left\langle A^{1 / 2} v, A^{1 / 2} u\right\rangle_{X}-a\left\langle A^{1 / 2} v, v\right\rangle_{X}\right. \\
& -a\left\langle A u, A^{1 / 2} u\right\rangle_{X}-2 a^{2} \operatorname{Re}\left\langle A^{1 / 2} v, A^{1 / 2} u\right\rangle_{X} \\
& \left.\quad-2 a^{2} i \operatorname{Im}\left\langle A^{1 / 2} v, A^{1 / 2} u\right\rangle_{X} \cdot\right\}
\end{aligned}
$$

Now use the fact that $\gamma_{1}^{1 / 2}\|x\|_{X}^{2} \leq\left\|A^{1 / 4} x\right\|_{X}^{2}$ for all $x \in \operatorname{dom} A^{1 / 2}$ to get

$$
\begin{aligned}
& \operatorname{Re}\langle\mathcal{A}(u, v),(u, v)\rangle_{e}= \frac{-a}{2\left(1-a^{2}\right)}\left\{\left\|A^{1 / 4} v\right\|_{X}^{2}+\left\|A^{3 / 4} u\right\|_{X}^{2}\right. \\
&\left.+2 a \operatorname{Re}\left\langle A^{1 / 4} v, A^{3 / 4} u\right\rangle_{X}\right\} \\
&= \frac{-a}{2\left(1-a^{2}\right)}\left\{(1-a)\left\|A^{1 / 4} v\right\|_{X}^{2}+(1-a)\left\|A^{3 / 4} u\right\|_{X}^{2}\right. \\
&\left.+a\left\|A^{1 / 4} v+A^{3 / 4} u\right\|_{X}^{2}\right\}
\end{aligned}
$$




$$
\begin{aligned}
& \leq \frac{-a \gamma_{1}^{1 / 2}}{2\left(1-a^{2}\right)}\left\{(1-a)\|v\|_{X}^{2}+(1-a)\left\|A^{1 / 2} u\right\|_{X}^{2}\right. \\
& \left.\quad+a\left\|v+A^{1 / 2} u\right\|_{X}^{2}\right\} \\
& =-a \gamma_{1}^{1 / 2}\|(u, v)\|_{e}^{2} .
\end{aligned}
$$

Also,

$$
\begin{aligned}
& \frac{\sqrt{1-a^{2}}}{a}\left(-\frac{\sqrt{1-a^{2}}}{a} \operatorname{Re}\langle\mathcal{A}(u, v),(u, v)\rangle_{e}-\left|\operatorname{Im}\langle\mathcal{A}(u, v),(u, v)\rangle_{e}\right|\right) \\
& \quad=\frac{1}{2 a}\left(\left\|A^{1 / 4} v\right\|_{X}^{2}+\left\|A^{3 / 4} u\right\|_{X}^{2}+2 a \operatorname{Re}\left\langle A^{1 / 4} v, A^{3 / 4} u\right\rangle_{X}\right. \\
& \left.\quad-2 \sqrt{1-a^{2}}\left|\operatorname{Im}\left\langle A^{1 / 4} v, A^{3 / 4} u\right\rangle_{X}\right|\right) \\
& \quad \geq 0
\end{aligned}
$$

This last inequality follows from the lemma below. Thus,

$$
\left|\operatorname{Im}\langle\mathcal{A}(u, v),(u, v)\rangle_{e}\right| \leq-\frac{\sqrt{1-a^{2}}}{a} \operatorname{Re}\langle\mathcal{A}(u, v),(u, v)\rangle_{e}
$$

and the result follows.

The following lemma, which applies in a general Hilbert space setting, was needed in the previous proof.

Lemma: Let $X$ be a Hilbert space, and $0<a<1$. Then

$$
\|x\|^{2}+\|y\|^{2}+2 a \operatorname{Re}\langle x, y\rangle-2 \sqrt{1-a^{2}}|\operatorname{Im}\langle x, y\rangle| \geq 0
$$

for all $x, y \in X$

Proof: Consider $f(\theta)=1+a \cos \theta-\sqrt{1-a^{2}}|\sin \theta|$. An elementary argument shows that $f(\theta) \geq 0$ for $0 \leq \theta \leq 2 \pi$, so that

$$
2 a \cos \theta-2 \sqrt{1-a^{2}}|\sin \theta| \geq-2 .
$$

Since $\langle x, y\rangle \in \mathbb{C}$, we have

$$
\operatorname{Re}\langle x, y\rangle=r \cos \theta, \operatorname{Im}\langle x, y\rangle=r \sin \theta, r=|\langle x, y\rangle| \geq 0, \text { and } 0 \leq \theta \leq 2 \pi .
$$

Thus

$$
\begin{aligned}
2 a \operatorname{Re}\langle x, y\rangle-2 \sqrt{1-a^{2}}|\operatorname{Im}\langle x, y\rangle| & =r\left(2 a \cos \theta-2 \sqrt{1-a^{2}}|\sin \theta|\right) \\
& \geq-2 r=-2|\langle x, y\rangle| \\
& \geq-2\|x\|\|y\|,
\end{aligned}
$$


by the Cauchy-Schwarz inequality. So

$$
\begin{aligned}
\|x\|^{2}+\|y\|^{2}+2 a \operatorname{Re}\langle x, y\rangle-2 \sqrt{1-a^{2}}|\operatorname{Im}\langle x, y\rangle| & \geq\|x\|^{2}+\|y\|^{2}-2\|x\|\|y\| \\
& =(\|x\|-\|y\|)^{2} \geq 0
\end{aligned}
$$

and the result follows.

Thus in our new inner product the numerical range $\Theta\left(\mathcal{A},\langle\cdot, \cdot\rangle_{e}\right)$ is equal to the convex hull of the eigenvalues of $\mathcal{A}$. (On a related note, it is straightforward to verify that in the new inner product the operator $\mathcal{A}$ is normal, which is not true in the energy inner product). It remains for us to define a new space $V_{e}$ and sesquilinear form $\sigma_{e}$. To this end, define $V_{e}=\operatorname{dom} A^{3 / 4} \times \operatorname{dom} A^{1 / 4}$ with norm

$$
\|(u, v)\|_{V_{e}}^{2}=\frac{1}{2\left(1-a^{2}\right)}\left\{\left\|A^{3 / 4} u\right\|_{X}^{2}+\left\|A^{1 / 4} v\right\|_{X}^{2}+2 a \operatorname{Re}\left\langle A^{3 / 4} u, A^{1 / 4} v\right\rangle_{X}\right\}
$$

and the obvious compatible inner product. Also define $\sigma_{e}: V_{e} \times V_{e} \rightarrow \mathbb{C}$ by

$$
\begin{aligned}
\sigma_{e}((u, v),(f, g))= & \frac{1}{2\left(1-a^{2}\right)}\left\{-a\left\langle A^{3 / 4} u, A^{3 / 4} f\right\rangle_{X}-a\left\langle A^{1 / 4} v, A^{1 / 4} g\right\rangle_{X}\right. \\
& \left.-\left\langle A^{3 / 4} u, A^{1 / 4} g\right\rangle_{X}+\left(1-2 a^{2}\right)\left\langle A^{1 / 4} v, A^{3 / 4} f\right\rangle_{X}\right\} .
\end{aligned}
$$

Without providing details, we observe that $\operatorname{dom} \mathcal{A} \subset V_{e} \subset H$, the embedding $V_{e} \subset H$ is compact, the sesquilinear form $\sigma_{e}$ is $V_{e}$-elliptic, and the operator $\mathcal{A}$ is associated with $\sigma_{e}$ via (2.1). As mentioned previously, one can now apply results for 'coercive' systems to (1.1). Instead, we finish by comparing Galerkin approximations defined using $\sigma_{e}$ and finite dimensional subspaces of $V_{e}$ versus those defined using $\sigma$ and subspaces of $V$. In particular we measure sensitivity of eigenvalues to unstructured perturbations by plotting the $\epsilon$-pseudospectrum of the matrix representations for the respective Galerkin schemes. Recall that for any $\epsilon>0$ the $\epsilon$-pseudospectrum of $A$ is the subset of the complex plane defined by (see [14] for details on definitions and results concerning pseudospectra)

$$
\Sigma_{\epsilon}(A)=\{\lambda \in \mathbb{C}: \lambda \in \Sigma(A+\Delta A) \text { for some } \Delta A \text { with }\|\Delta A\| \leq \epsilon\},
$$

where $\Sigma(A)$ is the spectrum of $A$. It is always true that $\Sigma(A) \subset \Sigma_{\epsilon}(A)$ and it is clear that the pseudospectra give a reasonable measure of sensitivity of eigenvalues to unstructured perturbations.

We consider a specific example using $X=L^{2}(0,1)$ and $A=\frac{d^{4}}{d x^{4}}$ in (1.1), which corresponds to a structurally damped Euler-Bernoulli beam with pinned boundary conditions. In Figure 1 we plot the 2-pseudospectrum of Galerkin approximation matrix representations $A^{N}$ in the energy norm for $N=8$ and $N=16$ subdivisions of $(0,1)$ and linear splines. In Figure 2 we do the same using the new inner product. However, for this case the pseudospectra are so well behaved that the 2-pseudospectrum cannot be distinguished from the 

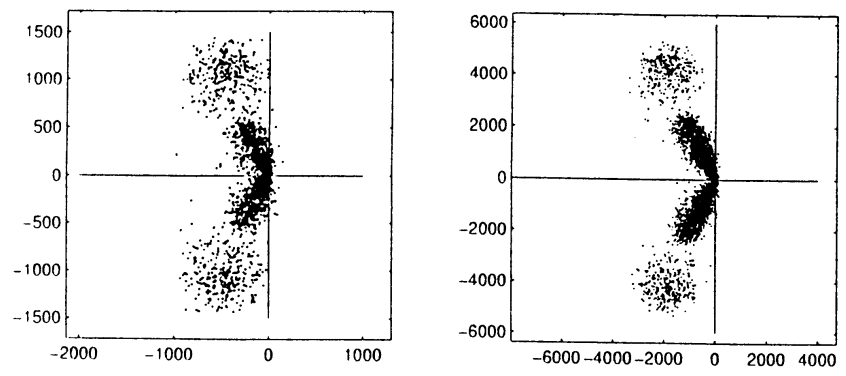

Figure 1 2-pseudospectrum of $A^{N}$ for $N=8$ and $N=16$

eigenvalues. So instead we plot the 75-pseudospectrum(!) - that is, the eigenvalues of the matrix representation $A_{e}^{N}$ (Galerkin approximation in the new inner product) are less sensitive to perturbations of magnitude 75 than are the eigenvalues of $A^{N}$ to perturbations of magnitude 2 .
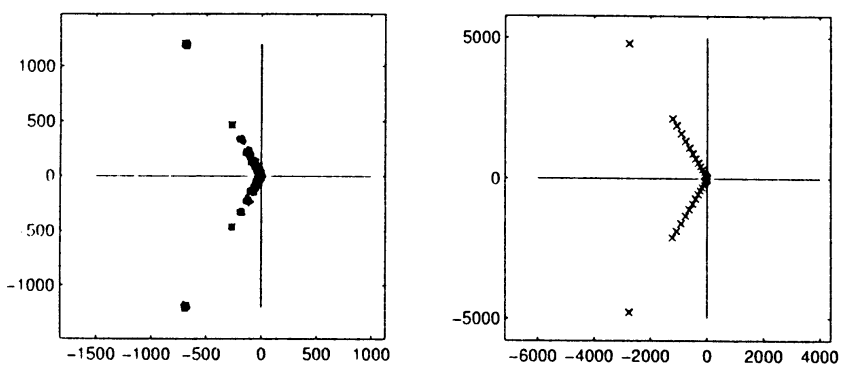

Figure 2 75-pseudospectrum of $A_{e}^{N}$ for $N=8$ and $N=16$

Finally, we point out that the model (1.1) has been considered in more generality in [3], [4], [7], and [10]. In particular, the operator $A^{1 / 2}$ in (1.1) has been replaced by $A^{\gamma}$ for $0<\gamma \leq 1$ or more generally by an operator $B$ 'similar' to $A^{\gamma}$. In [5] the ideas in this paper are considered in the more general case.

\section{References}

[1] H. T. Banks and K. Ito. A unified framework for approximation in inverse problems for distributed parameter systems. Control: Theory and Advanced Technology, 4(1):73-90, 1988. 
[2] H. T. Banks and K. Kunisch. The linear regulator problem for parabolic systems. SIAM J. Control and Optimization, 22:684-699, 1984.

[3] G. Chen and D. L. Russell. A mathematical model for linear elastic systems with structural damping. Quarterly of Applied Mathematics, 39:433-454, 1982.

[4] S. Chen and R. Triggiani. Proof of extensions of two conjectures on structural damping for elastic systems. Pacific Journal of Mathematics, 136:15$55,1989$.

[5] R. H. Fabiano. Coercive forms associated with elastic systems with damping. submitted.

[6] J. S. Gibson and A. Adamian. Approximation theory for linear quadratic Gaussian control of flexible structures. SIAM J. Control and Optimization, 29:1-37, 1991.

[7] F. Huang. On the mathematical model for linear elastic systems with analytic damping. SIAM Journal of Control and Optimization, 26:714724, 1988.

[8] T. Kato. Perturbation Theory for Linear Operators. Springer-Verlag, 1976.

[9] I. Lasiecka and R. Triggiani. Differential and Algebraic Riccati Equations with Applications to Boundary/Point Control Problems: Continuous Theory and Approximation Theory, volume 164 of Lecture Notes in Control and Information Sciences. Springer-Verlag, 1991.

[10] K. Liu and Z. Liu. Analyticity and differentiability of semigroups associated with elastic systems with damping and gyroscopic forces. Journal of Differential Equations, 141:340-355, 1997.

[11] A. Pazy. Semigroups of Linear Operators and Applications to Partial Differential Equations, volume 44 of Applied Mathematical Sciences. SpringerVerlag, 1983.

[12] R. E. Showalter. Hilbert Space Methods for Partial Differential Equations. Pitman, 1977. Out of print. A postscript file is available at http://ejde.math.swt.edu//mono-toc.html.

[13] H. Tanabe. Equations of Evolution. Pitman, 1979.

[14] L. N. Trefethen. Pseudospectra of linear operators. SIAM Review, 39:383406, 1997. 